\title{
The Anti-CD19 Antibody-Drug Conjugate Loncastuximab Tesirine
}

\author{
Krishna Goparaju, ${ }^{1}$ Allison Winter ${ }^{2,3}$ and Paolo F Caimi ${ }^{2,3}$
}

1. Department of Medicine, Division of Hematology and Oncology, University Hospitals Cleveland Medical Center, Cleveland, OH, USA;

2. Department of Hematology and Oncology, Cleveland Clinic, Cleveland, OH, USA; 3. Case Comprehensive Cancer Center, Cleveland, OH, USA

DOI: https://doi.org/10.17925/OHR.2021.17.2.95

7 he B-cell surface antigen CD19 was identified several decades ago as a valuable target for immunotherapy. Several antibody-based therapies targeting CD19 have shown efficacy in the treatment of B-cell malignancies, including Fc-engineered monoclonal antibodies, bispecific antibody constructs and chimeric antigen receptor T cells. Loncastuximab tesirine is an anti-CD19 antibody-drug conjugate that delivers a pyrrolobenzodiazepine dimer payload. Preclinical studies demonstrated that loncastuximab tesirine has activity against B-cell lymphoma in vitro and in vivo. The initial phase I trial of loncastuximab tesirine in relapsed/refractory (r/r) B-cell lymphomas included 183 patients, and although there were no dose-limiting toxicities, loncastuximab tesirine presented a specific pattern of adverse events, including oedema and effusions (31.7\%), elevation in gamma-glutamyl transferase without transaminase elevation (31.1\%) and rash (24.6\%). In a phase II trial conducted in $r / r$ diffuse large B-cell Iymphoma $(n=145)$, loncastuximab tesirine resulted in an overall response rate of $48.3 \%$ (24.1\% complete response) and median duration of response of 13.4 months. Outcomes were comparable among different disease risk groups, including elderly patients, and those with transformed indolent lymphoma and double-hit lymphoma. Given its single-agent activity and safety profile, loncastuximab tesirine is a valuable therapeutic alternative against B-cell malignancies. Multiple ongoing trials are investigating its role in combination regimens and other indications. This article reviews the development of loncastuximab tesirine and summarizes the results from published and ongoing trials.

\section{Keywords}

Non-Hodgkin lymphoma, immunotherapy, antibody-drug conjugate, anti-CD19 therapy, loncastuximab tesirine

Disclosures: Krishna Goparaju and Allison Winter have no financial or non-financial relationships or activities to declare in relation to this article. Paolo F Caimi reports research funding from ADC Therapeutics and Genentech, and consulting for ADC Therapeutics, Kite, Genentech, GenMab, Novartis, Epizyme, Incyte and Bristol Myers Squibb.

Review process: Double-blind peer review.

Compliance with ethics: This study involves a review of the literature and did not involve any studies with human or animal subjects performed by any of the authors.

Data availability: Data sharing is not applicable

to this article as no datasets were generated or analysed during the writing of this article.

Authorship: The named authors meet the Internationa Committee of Medical Journal Editors (ICMJE) criteria for authorship of this manuscript, take responsibility for the integrity of the work as a whole, and have given final approval for the version to be published. Access: This article is freely accessible at touchONCOLOGY.com @ Touch Medical Media 2021

Received: 7 September 2021

Accepted: 30 November 2021

Published online: 22 December 2021

Citation: touchREVIEWS in Oncology \& Haematology. 2021;17(2):95-100

Corresponding author: Paolo F Caimi, Cleveland

Clinic Lerner College of Medicine, Department of Hematology and Oncology, Cleveland Clinic Cleveland, OH, USA. E: caimip@ccf.org

Support: No funding was received in the publication of this article.
The addition of rituximab to chemotherapy provides an overall survival benefit in aggressive lymphomas, ${ }^{1}$ whereas for indolent lymphoma subtypes this addition has resulted in improvements in progression-free survival. ${ }^{2}$ For more than two decades, immunotherapy with monoclonal 'naked' antibodies targeting CD20 remained the primary representative of this therapeutic modality, ${ }^{3}$ with a small number of patients receiving anti-CD20 radioimmunotherapy. ${ }^{4}$

A number of immunotherapeutic agents have been developed in the past 5 years, most targeting new cell surface antigens. Targets include CD79a and CD19. CD79a is a central component of the B-cell receptor, which is targeted by the currently approved antibody-drug immunoconjugate, polatuzumab vedotin. ${ }^{5}$ CD19 is a 'pan B' surface marker present throughout B-cell maturation stages and has long been studied as a potential therapeutic target. ${ }^{6-8}$ Multiple approved agents targeting CD19 are now available, including chimeric antigen receptor (CAR) T cells, monoclonal antibodies (mAbs) and antibody-drug conjugates (ADCs). While all three therapeutic modalities are aimed at the same target, they have unique mechanisms of action, adverse safety profiles and indications

\section{CD19 as target in lymphoma Biology of CD19}

CD19 is a $95 \mathrm{kD}$ glycoprotein member of the immunoglobulin superfamily. It is composed of a highly conserved cytoplasmic domain, a single transmembrane domain and two extracellular C2 immunoglobulin-like domains separated by a small non-Ig-like helical region. ${ }^{9}$ The expression of CD19 throughout B-cell maturation stages, from late pro-B cells or early pre-B cells until loss of expression during terminal plasma cell differentiation, make it a reliable marker for normal and neoplastic B cells.9.10 Initial CD19 expression occurs at the time of immunoglobulin heavy chain gene rearrangement ${ }^{10,11}$ and is tightly regulated throughout $\mathrm{B}$-cell maturation, with mature $\mathrm{B}$ cells having 3-fold higher expression, 9 reported to range between 10,000 and 100,000 sites per cell. ${ }^{12,13}$ CD19 forms part of a multimolecular signalling complex in the surface of the B cell along with CD21, the tetraspanin membrane protein CD81, and CD225.9.10,14 Additionally, CD19 modulates/ amplifies B-cell receptor-dependent and -independent signals through activation of Src family kinases as well as PI3K and AKT.

Once mAbs (or antibody conjugates) bind to B-cell surface antigens, they undergo internalization and trafficking to lysosomes, where the antibody is degraded and a conjugated payload (toxin, cytotoxic agent or radioisotope) is released into the cytoplasm. ${ }^{12,15}$ Press et al. described several 
characteristics of mAbs relevant for the choice of 'antibody-directed immunotherapy', including target antigen density and tissue specificity, antibody immunoreactivity, avidity, isotype, rate of degradation and intracellular routing. ${ }^{12}$ Perhaps persistence of surface antigen should be included as a relevant characteristic. Anti-CD19 antibodies were noted at the time to have high antigen density (although lower than anti-CD20), rapid internalization (slower than anti-CD22 and faster than anti-CD20) and adequate internal routing and antigen persistence. These characteristics suggested CD19 as a valuable target for antibodybased immunotherapy.

\section{Immunotherapy targeting CD19}

\section{Initial studies}

Early studies with mAbs targeting CD19 for treatment of B-cell malignancies showed limited clinical activity as single agents ${ }^{16}$ or in combination with interleukin-2. ${ }^{17}$ Subsequently, an antibody-toxin conjugate of anti-B4-blocked ricin (anti-B4-BR) as post autologous stem cell transplant maintenance for patients in complete remission showed that 23/31 infused patients developed human anti-mouse or anti-ricin antibodies and a continued relapse pattern was observed. ${ }^{18}$

\section{Optimized monoclonal antibodies}

Subsequent developments of immunotherapy included the development of Fc-engineered mAbs, CAR T cells and ADCs. Among Fc-engineered mAbs, inebilizumab (MEDI-551) is a humanized, affinity and afucosylated IgG1 kappa mAb targeting CD19, with enhanced antibody-dependent cellular cytotoxicity (ADCC). ${ }^{19}$ Phase I results suggested responses in aggressive and indolent lymphomas, ${ }^{20}$ but combination studies and randomized phase II trials showed limited activity. ${ }^{6}$ Tafasitamab (MOR208, $\mathrm{XmAb5574)}$ is an Fc-engineered domain with improved binding to $\mathrm{FC} \gamma$ receptors ${ }^{21}$ and increased $A D C C .{ }^{21,22}$ Single-agent activity of tafasitamab against aggressive and indolent lymphomas was limited (overall response rate [ORR] for diffuse large B-cell lymphoma [DLBCL] 26\%, follicular lymphoma [FL] 30\%, chronic lymphocytic leukaemia 22\%) and consisted mostly of partial responses (PR). ${ }^{23}$ L-MIND (A Study to Evaluate the Safety and Efficacy of Lenalidomide With MOR00208 in Patients With R-R DLBCL; ClinicalTrials.gov identifier: NCT02399085) was a phase II trial evaluating the combination of tafasitamab and lenalidomide in relapsed or refractory $(r / r) D L B C L$ in transplant-ineligible patients. The combination resulted in an ORR of $60 \%$, with a complete response (CR) in $43 \%$ and a median duration of response of 43.9 months. ${ }^{24,25}$ These results led to regulatory approval of tafasitamab in combination with lenalidomide for treatment of patients with $r / r$ DLBCL after $\geq 1$ line of therapy who are ineligible for autologous stem cell transplant.

\section{Bispecific antibody constructs}

Bispecific antibody constructs correspond to a large group of molecules that includes immune molecules capable of recognizing two distinct epitopes. ${ }^{26}$ The formats can be variable in size and structure, from small proteins with linked antigen-binding fragments, to large multidomain proteins. While antigen selection may be varied, the bispecific agents targeting CD19 are mostly T-cell engagers, targeting CD3 as the second epitope. ${ }^{26}$ Blinatumomab is a bispecific T-cell engager that is approved for the treatment of CD19-positive acute lymphoblastic leukaemia. In the initial phase I trial of blinatumomab, neurological toxicity was common (all grades $71 \%$, grade $322 \%$ ). Among patients treated at the maximum tolerated dose $\left(60 \mu \mathrm{g} / \mathrm{m}^{2} /\right.$ day), responses were observed across several lymphoma subtypes (FL 12/15, DLBCL 6/11, mantle cell lymphoma [MCL] 5/7) although, again, most were PRs. ${ }^{27}$ In a subsequent phase II trial, dose increase and steroid premedication over the course of 3 weeks permitted escalation of blinatumomab to higher doses $\left(112 \mu \mathrm{g} / \mathrm{m}^{2} /\right.$ day $)$, with lower incidence of neurological toxicity. In addition to neurological toxicity, the biggest challenge of blinatumomab is its pharmacokinetic profile, with a very short half-life requiring continuous infusion for 4-8 weeks. Moreover, dose increases or re-initiation of infusion after an interruption require inpatient monitoring. These logistical challenges have limited further development of blinatumomab in non-Hodgkin lymphomas.

\section{Chimeric antigen receptor T cells}

CAR T cells are cells that have been genetically modified to express a chimeric receptor that contains an extracellular B-cell antigen recognition with transmembrane and intracellular domains than mediate T-cell activation. Four anti-CD19 CAR T-cell therapies are currently approved for treatment of $r / \mathrm{r}$ B-cell malignancies: axicabtagene ciloleucel for $\mathrm{DLBCL}$ and $\mathrm{FL}^{28}$ tisagenlecleucel for acute lymphoblastic leukaemia and $\mathrm{DLBCL},{ }^{29,30}$ lisocabtagene maraleucel for $\mathrm{DLBCL}^{31}$ and brexucabtagene autoleucel for MCL. ORRS in DLBCL range between 52\% and 83\%, with $\mathrm{CR}$ rates of $40-54 \%$. Long-term remissions are achieved in approximately $40 \%$ of $r / r$ DLBCL cases. The adverse event pattern of these adoptive immune therapies appears to be consistent, with acute immunoinflammatory toxicities including cytokine release syndrome and immune effector-associated neurotoxicity syndrome being the predominant complications. Prolonged cytopenia, hypogammaglobulinaemia and opportunistic infections have also been reported. ${ }^{28-33}$ The incidence of adverse events appears to be slightly different between CART constructs containing CD28 versus 4-1BB co-stimulatory signals.

\section{Antibody-drug conjugates targeting CD19}

ADCs are antibodies bound to other molecules through a chemical linker. The 'payload' can include small potent cytotoxic molecules, protein toxins, immunomodulatory proteins, radionuclides and others. ADCs can be considered site-specific prodrugs. ${ }^{34}$ As discussed above, the internalization kinetics of CD19 make it an attractive target for these agents, ${ }^{12}$ as internalization and lysosomal degradation are the mechanisms through which the payload is released to the target cell population.

Several ADCs targeting CD19 have been evaluated in clinical trials, including coltuximab ravtansine, denintuzumab mafodotin, SGNCD19B and loncastuximab tesirine. Coltuximab ravtansine (SAR3419) and denintuzumab mafodotin (SGN-CD19A) were ADCs conjugated to antimicrotubule agents, specifically a maytansinoid and monomethyl auristatin F. Both agents were associated with ocular toxicity: $44 \%$ for coltuximab and $65 \%$ for denintuzumab. ${ }^{35}$ Blurred vision was the most common manifestation and corneal epitheliopathy was the main ocular finding. ${ }^{35} \mathrm{~A}$ phase II trial of coltuximab failed to meet its primary endpoint, achieving responses in only $31 \%$ of patients, most of them PRs. ${ }^{36}$ Further development of both agents has been discontinued. ${ }^{37}$ Other anti-CD19 activity in preclinical models includes SGN-CD19B (pyrrolobenzodiazepine [PBD] payload), HuB44-DGN452 (alkylating agent payload) ${ }^{38}$ and CD19thiomab-Amanitin (RNA polymerase II inhibitor payload). ${ }^{6,39}$

\section{Loncastuximab tesirine}

Loncastuximab tesirine is an ADC comprising SG3199, a PBD dimer, conjugated to a humanized IgG1 anti-CD19 mAb through a valine-alanine protease cleavable linker.

PBD as a monomer is derived from Actinomyces and has potent alkylating properties. ${ }^{40}$ Early ADC development conjugated a synthetic PBD dimer payload, talirine (SGD-1910), with a CD33 mAb for the treatment of acute myeloid leukemia. ${ }^{41}$ Tesirine (SG3249) was developed through the addition of a PEG8 spacer, incorporation of N10 nitrogen in the cleavable valinealanine prodrug linker, and substitution of $\mathrm{C} 2$ aryls to methyl groups 


\section{Figure 1: Mechanism of action of loncastuximab tesirine}

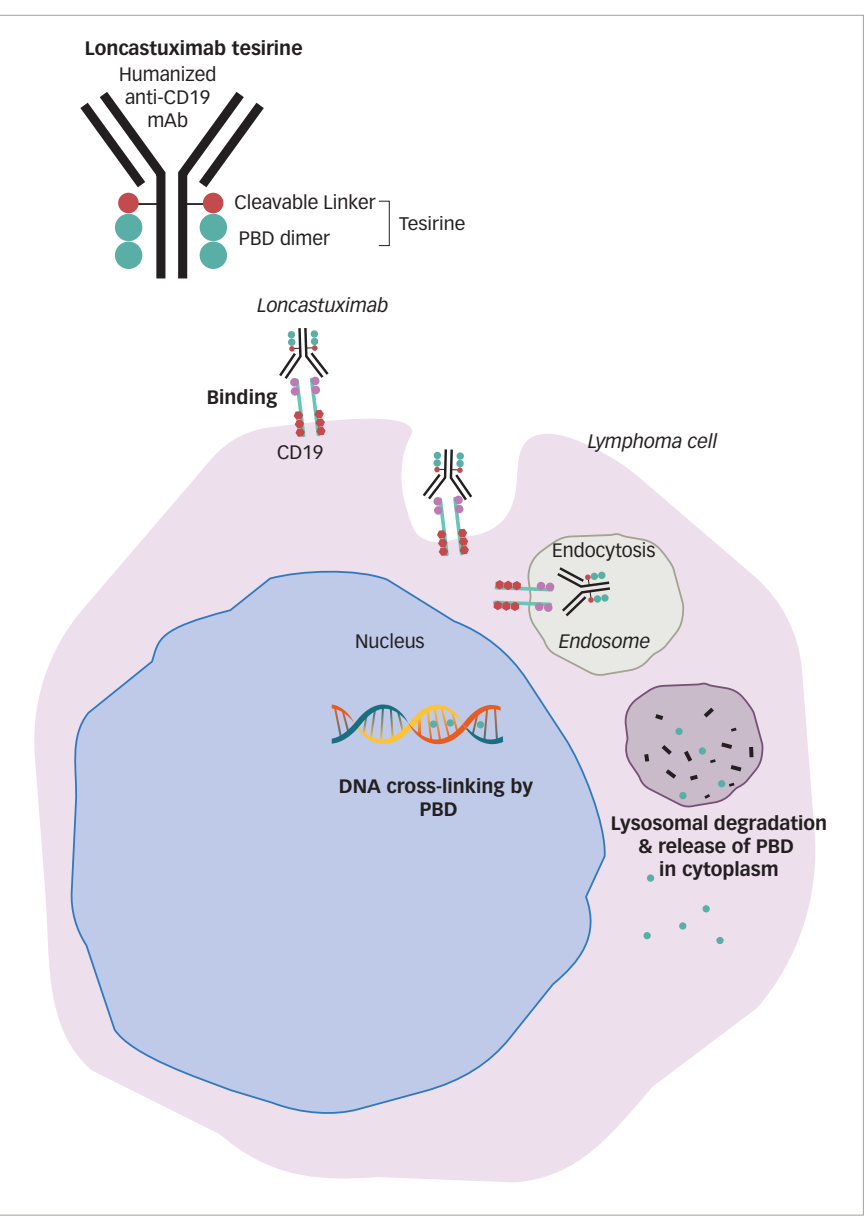

$m A b=$ monoclonal antibody $; P B D=$ pyrrolobenzodiazepine.

in the talirine structure. These modifications were aimed at increasing solubility without decreasing potency. The substitution of the C2 aryls with methyl groups allowed for more flexible and discrete inter-strand DNA binding that evades the host DNA repair mechanisms. ${ }^{40,42}$

Preclinical studies show that loncastuximab tesirine is internalized after binding to surface CD19 followed by release of the PBD payload through lysosomal cleavage of the protease linker, resulting in neoplastic cell cytotoxicity (Figure 1). ${ }^{42,43}$ Animal studies measured the cytotoxic effects on CD19+/CD19- tumours after administering free SG3199 warhead and Ioncastuximab tesirine. The preclinical studies showed dose-dependent antitumour activity of Ioncastuximab tesirine. In addition, the investigators observed bystander killing of CD19-negative cells, considered helpful in targeting tumours with decreased expression of CD19. ${ }^{43}$

\section{Clinical trials}

A single-arm, multicentre phase I trial with single-agent loncastuximab tesirine included 183 patients with r/r B-cell lymphoma (Table 1). ${ }^{44,45}$ The dose-escalation phase included 88 patients and the dose-expansion phase included 95 patients. The diagnoses included DLBCL ( $n=139)$, FL ( $n=14)$, $\operatorname{MCL}(n=15)$ and other histologies $(n=15)$. The median number of prior therapies was 3. Loncastuximab tesirine was given intravenously over 60 minutes on Day 1 of each 21-day cycle at doses of 15-200 $\mu \mathrm{g} / \mathrm{kg}$. No doselimiting toxicities were observed and none of the dose levels met maximum tolerated dose criteria. However, cumulative toxicity was observed at $200 \mu \mathrm{g} / \mathrm{kg}$, with higher incidence of treatment-emergent adverse events (TEAES) compared with the 120 and $150 \mu \mathrm{g} / \mathrm{kg}$ cohorts. These two levels were selected for the dose-expansion cohorts $(120 \mu \mathrm{g} / \mathrm{kg} \mathrm{n}=26 ; 150 \mu \mathrm{g} /$ $\mathrm{kg} \mathrm{n=69).} \mathrm{The} \mathrm{median} \mathrm{number} \mathrm{of} \mathrm{loncastuximab} \mathrm{tesirine} \mathrm{cycles} \mathrm{was} 2$ (range 1-24), with a median dose per cycle of $129.9 \mu \mathrm{g} / \mathrm{kg}$. The median duration of treatment was 64 days (range 22-532 days). A total of 181 patients (98.3\%) experienced at least one TEAE. Haematological toxicity was common, including thrombocytopenia, anaemia and neutropenia. Among non-haematological TEAEs, the most common were fatigue $(n=78,42.6 \%)$, nausea $(n=59,32.2 \%)$, peripheral oedema $(n=58,31.7 \%)$ and elevated gamma-glutamyl transferase (GGT; $n=57,31.1 \%$ ). Cutaneous reactions were also common, including rash in 45 patients (24.6\%), most frequently in sun-exposed areas. Oedema and effusions occurred in $86(47.0 \%)$, including both peripheral oedema $(n=58,31.7 \%)$ and pleural effusions ( $n=39,21.3 \%$ ). The incidence of oedema and effusions decreased after the introduction of corticosteroid premedication. Grade 3 and higher TEAEs were observed in $77 \%$ of patients. The most frequent grade $\geq 3$ adverse events were haematological, followed by elevated GGT and electrolyte abnormalities. Progressive disease was the primary cause of treatment discontinuation ( $n=83,45.4 \%$ ).

The ORR in the evaluable population ( $n=180$ ) was $45.6 \%$ ( $95 \%$ confidence interval 38.1-53.1\%), with $26.7 \%$ showing a CR. A dose-response relationship was observed, with ORRs of $29.4 \%$ for patients treated with doses of $15-90 \mu g / k g$ compared with $47.2 \%$ for patients treated at doses of $120-200 \mu g / k g$.

Immunogenicity analysis found anti-drug antibodies were infrequent and with low or very low $\log _{2}$ titres, suggesting loncastuximab tesirine did not induce the formation of anti-drug antibodies.

LOTIS-2 (Study to Evaluate the Efficacy and Safety of Loncastuximab Tesirine in Patients With Relapsed or Refractory Diffuse Large B-Cell Lymphoma; ClinicalTrial.gov identifier NCT03589469) was a multicentre, phase II trial that enrolled patients with $r / r$ DLBCL after two or more prior lines of therapy (Table 1). ${ }^{46}$ Loncastuximab tesirine was given intravenously every 3 weeks with an initial loading dose of $150 \mu \mathrm{m} / \mathrm{kg}$ for the first two cycles, then $75 \mathrm{\mu g} / \mathrm{kg}$ for subsequent cycles for a maximum of 12 months. A total of 145 patients were enrolled. The median number of cycles was 4 . ORR was $48.3 \%$, with $24.1 \%$ of patients achieving CR. ${ }^{46}$ The median duration of response for the entire population was 13.4 months. Subgroup analyses showed comparable response rates and duration of response in specific subgroups, including elderly patients, and those with high-grade lymphoma with MYC and BCL-2 and/or $B C L-6$ rearrangements, transformed indolent lymphoma, and primary refractory disease. ${ }^{47}$ The primary causes of treatment discontinuation were progressive disease and treatment intolerance. The most frequent all-grade TEAEs included elevated GGT (40.7\%), neutropaenia (39.3\%), thrombocytopaenia (33.1\%), fatigue (27.6\%) and anaemia (26.2\%). Among grade $\geq 3$ TEAEs, the most common were neutropaenia (25.5\%), thrombocytopaenia (17.9\%), elevated GGT (16.6\%) and anaemia (10.3\%). Tolerability was similar among all age groups. ${ }^{47}$

\section{Ongoing combination studies}

The single-agent activity and specific safety profile of Ioncastuximab tesirine make it an attractive agent for combination with other drugs. LOTIS-3 is a phase I/II trial (NCT03684694) evaluating the safety and recommended dose and schedule of loncastuximab tesirine in combination with the Bruton's tyrosine kinase inhibitor, ibrutinib. ${ }^{48}$ The target population includes patients with $r / r$ DLBCL and MCL. The dose-escalation phase of the study is complete. The dosing on the expansion phase includes loncastuximab tesirine given in cycles 1 and 2 at a dose of $60 \mu \mathrm{g} / \mathrm{kg}$, followed by ibrutinib alone in cycles 3 
Table 1: Characteristics and results of phase I and phase II trials of loncastuximab tesirine

\begin{tabular}{|c|c|c|c|c|}
\hline & \multicolumn{2}{|l|}{ LOTIS $1^{44,45}$} & \multicolumn{2}{|l|}{ LOTIS $2^{46,47}$} \\
\hline Study phase & \multicolumn{2}{|l|}{ । } & \multicolumn{2}{|l|}{$\|$} \\
\hline \multicolumn{5}{|l|}{ Study population } \\
\hline Number of patients & \multicolumn{2}{|l|}{183} & \multicolumn{2}{|l|}{145} \\
\hline Age, median (range), years & \multicolumn{2}{|l|}{63 (range $20-87$ ) } & \multicolumn{2}{|l|}{66 (IQR 56-71) } \\
\hline \multicolumn{5}{|l|}{ Diagnoses, n } \\
\hline DLBCL & \multicolumn{2}{|l|}{139} & \multicolumn{2}{|l|}{127} \\
\hline $\mathrm{HGBCL-DH/TH}$ & \multicolumn{2}{|l|}{23} & \multicolumn{2}{|l|}{15} \\
\hline Transformed indolent & \multicolumn{2}{|l|}{37} & \multicolumn{2}{|l|}{29} \\
\hline HGBCL, NOS & \multicolumn{2}{|l|}{-} & \multicolumn{2}{|l|}{11} \\
\hline Mantle cell lymphoma & \multicolumn{2}{|l|}{15} & \multicolumn{2}{|l|}{-} \\
\hline Follicular lymphoma & \multicolumn{2}{|l|}{14} & - & \\
\hline Chronic lymphocytic leukaemia & 6 & & - & \\
\hline Marginal zone lymphoma & 6 & & - & \\
\hline PMBCL & 1 & & 7 & \\
\hline Other & 2 & & - & \\
\hline Number of prior lines of therapy, median (range) & 3 (range 1-13) & & $3($ (IQR 2-4) & \\
\hline Primary refractory, $\mathrm{n}$ & 43 & & 29 & \\
\hline Prior HCT, n & 42 & & 24 & \\
\hline Prior CAR T, n & 3 & & 13 & \\
\hline Disease response & & & & \\
\hline Overall response, \% (95\% Cl) & $45.6(38.1-53.1)$ & & $48.3(39.9-56.7)$ & \\
\hline Complete response, \% (95\% Cl) & 26.7 & & $24.1(17.4-31.9)$ & \\
\hline Safety & & & & \\
\hline Adverse event, n (\%) & All grade (\%) & Grade $\geq 3(\%)$ & All grades (\%) & Grade $\geq 3(\%)$ \\
\hline Haematologic toxicity & & & & \\
\hline Anaemia & $60(32.8)$ & $28(15.3)$ & $38(26)$ & $15(10)$ \\
\hline Neutropenia & $106(59.2)$ & $71(39.7)$ & $34(24)$ & $14(10)$ \\
\hline Thrombocytopenia & $128(71.1)$ & $48(26.7)$ & $40(27)$ & $18(12)$ \\
\hline Lymphopenia & - & $12(6.6)$ & $6(4)$ & $3(2)$ \\
\hline Febrile neutropenia & - & $10(5.5)$ & $5(3)$ & $5(3)$ \\
\hline Non-haematologic toxicity & & & & \\
\hline Fatigue & $78(42.6)$ & & $40(27)$ & $2(1)$ \\
\hline Nausea & $59(32.2)$ & & $34(23)$ & - \\
\hline Oedema peripheral & $58(31.7)$ & & $29(20)$ & $2(1)$ \\
\hline GGT increased & 57 (31.1) & $39(21.3)$ & $59(41)$ & $24(16)$ \\
\hline Rash & $45(24.6)$ & & $19(13)$ & $1(1)$ \\
\hline Dyspnoea & $41(22.4)$ & & 17 & $2(1)$ \\
\hline Constipation & $40(21.9)$ & & $17(12)$ & - \\
\hline Pleural effusion & $39(21.3)$ & & $15(10)$ & $3(2)$ \\
\hline Blood ALP increased & $37(20.2)$ & $12(6.6)$ & $29(20)$ & $1(1)$ \\
\hline AST increased & $34(18.6)$ & & $23(16)$ & $1(1)$ \\
\hline Decreased appetite & $34(18.6)$ & & $22(15)$ & \\
\hline Cough & $34(18.6)$ & & $32(22)$ & $1(1)$ \\
\hline Pyrexia & $33(18.0)$ & & $28(19)$ & \\
\hline Vomiting & 32 (17.5) & & $19(13)$ & - \\
\hline ALT increased & $32(17.5)$ & & $23(16)$ & $4(3)$ \\
\hline Abdominal pain & 29 (15.8) & & $16(11)$ & $4(3)$ \\
\hline Diarrhoea & $28(15.3)$ & & $22(15)$ & $3(2)$ \\
\hline Hypokalaemia & $23(12.6)$ & $10(5.5)$ & $20(15)$ & $6(4)$ \\
\hline Erythema & $21(11.5)$ & & $15(11)$ & \\
\hline Pruritus & 20 (10.9) & & $18(12)$ & \\
\hline
\end{tabular}




\begin{tabular}{|c|c|c|c|c|}
\hline & \multicolumn{2}{|l|}{ LOTIS $1^{44,45}$} & \multicolumn{2}{|l|}{ LOTIS $2^{46,47}$} \\
\hline \multicolumn{5}{|l|}{ Safety } \\
\hline Adverse event, n (\%) & All grade (\%) & Grade $\geq 3(\%)$ & All grades (\%) & Grade $\geq 3(\%)$ \\
\hline Dizziness & $20(10.9)$ & & & \\
\hline Rash maculopapular & 19 (10.4) & & $8(6)$ & $1(1)$ \\
\hline Hyperglycaemia & 19 (10.4) & & & \\
\hline Hypocalcaemia & & & $12(9)$ & $1(1)$ \\
\hline Photosensitivity & & & $15(10)$ & $3(2)$ \\
\hline Insomnia & & & $16(11)$ & \\
\hline Headache & & & $15(11)$ & $1(1)$ \\
\hline
\end{tabular}

$A L P=$ alkaline phosphatase; $A L T=$ alanine transaminase; $A S T=$ aspartate transaminase; $C A R T=$ chimeric antigen receptor $T$ cells; $C l=$ confidence interval; $D H / T H=$ double hit/triple hit with rearrangements of $M Y C$ and $B C L-2$ and/or $B C L-6 ; D L B C L=$ diffuse large $B$-cell lymphoma; GGT = gamma-g/utamyl transferase; $H C T=$ hematopoietic cell transplantation; $\mathrm{HGBCL}=$ high-grade $B$-cell lymphoma; $I Q R=$ interquartile range; NOS = not otherwise specified; $P M B C L=$ primary mediastinal $B$-cell lymphoma.

and 4. Loncastuximab is then repeated in cycles 5 and 6 in responding patients. Ibrutinib is given at a dose of $560 \mathrm{mg}$ for up to 1 year. In a recent update, 37 patients were enrolled (30 DLBCL, $7 \mathrm{MCL}$ ). The median number of prior therapies was 2 (range 1-6) and 18 patients were refractory to the last line of treatment. The median number of cycles of loncastuximab tesirine and ibrutinib were 2 (range 1-4) and 4 (range 1-14), respectively. Median treatment duration was 105 days (range 18-379). All patients experienced TEAES, with haematological toxicity including thrombocytopaenia (29.7\%) and anaemia (24.3\%) being most common. Non-haematological toxicity included fatigue, rash and diarrhoea (all 21.6\%). Grade $\geq 3$ events occurred in 24 patients (64.9\%), most frequently haematological in nature. The ORR and $\mathrm{CR}$ rate were $63.9 \%$ and $36.1 \%$, respectively. This study is ongoing with enrolment currently in the phase II portion.

\section{Upcoming trials}

LOTIS-5 (Study to Evaluate Loncastuximab Tesirine With Rituximab Versus Immunochemotherapy in Participants With Relapsed or Refractory Diffuse Large B-Cell Lymphoma; ClinicalTrials.gov indentifer: NCT04384484) is a phase III, randomized controlled study enrolling patients with $r / r$ DLBCL and comparing loncastuximab tesirine plus rituximab versus rituximab, gemcitabine and oxaliplatin. Estimated enrolment is 350 patients. ${ }^{49}$ An investigator-initiated phase $\|$ trial is evaluating the combination of Ioncastuximab tesirine and rituximab for treatment of $\mathrm{r} / \mathrm{r}$ FL (NCT04998669), ${ }^{50}$ whereas LOTIS-6 (Study to Evaluate the Efficacy and Safety of Loncastuximab Tesirine Versus Idelalisib in Participants With Relapsed or Refractory Follicular Lymphoma; ClinicalTrials.gov identifier: NCT04699461) will be a randomized phase II trial comparing loncastuximab tesirine and idelalisib for treatment of patients with r/r FL. ${ }^{51}$ LOTIS-7 (A Study to Evaluate the Safety and Anticancer Activity of Loncastuximab Tesirine in Combination With Other Anti-cancer Agents in Participants With Relapsed or Refractory B-cell Non-Hodgkin Lymphoma; ClinicalTrials.gov identifer: NCT04970901) is a phase $\mathrm{lb}$ trial evaluating the combination of loncastuximab tesirine with other agents, including gemcitabine, lenalidomide, umbralisib and polatuzumab vedotin for treatment of relapsed B-cell lymphomas. ${ }^{52}$ Finally, the safety of the addition of Ioncastuximab tesirine to rituximab plus cyclophosphamide, doxorubicin hydrochloride, vincristine, prednisolone (R-CHOP) is being evaluated in the phase Ib LOTIS-8 trial (A Study to Evaluate the Tolerability, Safety, Pharmacokinetics, and Antitumor Activity of Loncastuximab Tesirine in Combination With Rituximab, Cyclophosphamide, Doxorubicin, Vincristine, and Prednisone (R-CHOP) in Participants With Previously Untreated Diffuse Large B-cell Lymphoma; ClinicalTrials.gov identifier: NCT04974996). ${ }^{53}$

\section{Conclusions}

Decades after the identification of CD19 as a potential therapeutic target in B-cell malignancies, multiple antibody-based therapies targeting CD19 have demonstrated safety and efficacy. Loncastuximab tesirine is the first anti-CD19 ADC to receive regulatory approval. It is also the first approved ADC to deliver a novel class of payload, a PBD dimer. The safety profile of loncastuximab tesirine differs from other approved ADCs and other CD19-targeting agents: it is characterized by the absence of peripheral neuropathy, cytokine release syndrome and immune effectorassociated neurotoxicity syndrome; however, it is associated with peripheral oedema, effusions, photosensitivity and elevated GGT. The efficacy against DLBCL appears to be consistent throughout several risk groups. Very limited data suggest loncastuximab tesirine can be used prior to or after CAR T cell therapy, ${ }^{54}$ but more extensive research needs to validate the sequencing of anti-CD19 agents.

The safety profile, single-agent activity and administration schedule suggest that loncastuximab tesirine could be a valuable addition to combination therapy regimens for B-cell lymphomas. Early data from a single, phase I study suggest that loncastuximab tesirine can be effectively combined with ibrutinib, but ongoing and future studies should continue to explore both additional combinations and indications for this agent. $\square$
1. Coiffier $B$, Lepage $E$, Brière J, et al. CHOP chemotherapy plus rituximab compared with $\mathrm{CHOP}$ alone in elderly patients with diffuse large-B-cell lymphoma. New Eng/ J Med. 2002;346:235-42.

2. Lumish M, Falchi L, Imber BS, et al. How we treat mature B-cell neoplasms (indolent B-cell lymphomas). J Hematol Oncol. 2021;14:5.

3. Feugier P. A review of rituximab, the first anti-CD20 monoclonal antibody used in the treatment of B non-Hodgkin's lymphomas. Future Oncol. 2015;11:1327-42.

4. Read ED, Eu P, Little PJ, Piva TJ. The status of

radioimmunotherapy in $\mathrm{CD} 20+$ non-Hodgkin's lymphoma. Target radioimmunotherapy

5. Sehn $\mathrm{LH}$, Herrera AF, Flowers $\mathrm{CR}$, et al. Polatuzumab vedotin in relapsed or refractory diffuse large B-cell lymphoma. J Clin Oncol. 2020;38:155-65

6. Watkins MP, Bartlett NL. CD19-targeted immunotherapies fo treatment of patients with non-Hodgkin B-cell lymphomas. Expert Opin Investig Drug. 2018;27:601-11.

7. Scheuermann RH, Racila E. CD19 antigen in leukemia and Iymphoma diagnosis and immunotherapy. Leuk Lymphoma. 2009;18:385-97.

8. Hammer O. CD19 as an attractive target for antibody-based therapy. MAbs. 2012;4:571-7.

9. Wang K, Wei G, Liu D. CD19: a biomarker for B cell development lymphoma diagnosis and therapy. Exp Hematol Oncol. 2012;1:36.
10. Tedder TF. CD 19: a promising B cell target for rheumatoid arthritis. Nat Rev Rheumatol. 2009;5:572-7.

11. Anderson KC, Bates MP, Slaughenhoupt BL, et al. Expression of human B cell-associated antigens on leukemias and lymphomas: a model of human B cell differentiation. Blood. 1984;63:1424-33.

12. Press $\mathrm{OW}$, Farr $\mathrm{AG}$, Borroz $\mathrm{Kl}$, et al. Endocytosis and degradation of monoclonal antibodies targeting human B-cell malignancies. Cancer Res. 1989:49:4906-12.

13. Du X, Beers R, FitzGerald DJ, Pastan I. Differential cellular internalization of anti-CD19 and -CD22 immunotoxins results in different cytotoxic activity. Cancer Res. 2008;68:6300-5.

14. Nagro CJD, Otero DC, Anzelon AN, et al. CD 19 function in 
central and peripheral B-cell development. Immunol Res. 2005;31:119-31.

15. Li Z , Wang M, Yao X, et al. Development of novel anti-CD19 antibody-drug conjugates for B-cell lymphoma treatment. Int Immunopharmacol. 2018:62:299-308.

16. Hekman A, Honselaar A, Vuist WMJ, et al. Initial experience with treatment of human B cell lymphoma with anti-CD19 monoclonal antibody. Cancer Immunol Immunother. 1991;32:364-72

17. Vlasveld LT, Hekman A, Vyth-Dreese FA, et al. Treatment of low-grade non-Hodgkin's lymphoma with continuous infusion of low-dose recombinant interleukin-2 in combination with the B-cell-specific monoclonal antibody CLB-CD19. Cancer Immuno Immunother. 1995;40:37-47.

18. Grossbard ML, Multani PS, Freedman AS, et al. A Phase II study of adjuvant therapy with anti-B4-blocked ricin after autologous bone marrow transplantation for patients with relapsed B-Cel non-Hodgkin's lymphome. Clin Cancer Res. 1999-5.2392-8.

19. Gallaghers Turman S Yusuft et al Pharmacological profie Gallager of MEDI-551, a novel anti-CD19 antibody, in human CD19

20. Forero-Torres A, Hamadani M, Fanale MA, et al. Safety profile . Forero-Torres A, Hamadani M, Fanale MA, et al. Safety profile
and clinical response to MEDI-551, a humanized monoclonal anti-CD19, in a Phase $1 / 2$ study in adults with relapsed or refractory advanced B-cell malignancies. Blood. 2013;122:1810.

21. Horton HM, Bernett MJ, Pong E, et al. Potent in vitro and in vivo activity of an Fc-engineered anti-CD19 monoclonal antibody against Iymphoma and leukemia. Cancer Res. 2008;68:8049-57.

22. Kellner C, Zhukovsky EA, Pötzke A, et al. The Fc-engineered CD19 antibody MOR208 (XmAb5574) induces natural killer cell-mediated lysis of acute lymphoblastic leukemia cells from pediatric and adult patients. Leukemia. 2013;27:1595-8.

23. Jurczak W, Zinzani PL, Gaidano G, et al. Phase lla study of the CD19 antibody MOR208 in patients with relapsed or refractory B-cell non-Hodgkin's lymphoma. Ann Oncol. 2018;29:1266-72.

24. Salles G, Duell J, Barca EG, et al. Tafasitamab plus lenalidomide in relapsed or refractory diffuse large B-cell lymphoma (L-MIND) a multicentre, prospective, single-arm, phase 2 study. Lancet Oncol. 2020:21:978-88.

25. Düll J, Maddocks KJ, Gonzalez-Barca E, et al. Long-term analyses from L-MIND, a phase II study of tafasitamab (MOR208) combined with lenalidomide (LEN) in patients with relapsed or refractory diffuse large B-cell lymphoma (R/R DLBCL). I Clin Oncol. 2021;39:7513.

26. Labrijn AF, Janmaat ML, Reichert JM, Parren PWHI. Bispecific antibodies: a mechanistic review of the pipeline. Nat Rev Drug Discov. 2019;18:585-608.

27. Goebeler M-E, Knop S, Viardot A et al. Bispecific T-cell engage (BiTE) antibody construct blinatumomab for the treatment of patients with rapsed/refractory non-Hodgkin lymphomt of patients with clapsed/refractory non Hodgkin lymphoma: fin

esults from a Phase Is sudy JClin Oncol. 2016:34:1104-11.

8. Neelapu SS, Locke FL, Bartlett NL, et al. Axicabtagene ciloleuce CAR T-cell therapy in refractory large B-cell lymphoma. New Eng J Med. 2017:377:2531-44.
29. Schuster SJ, Svoboda J, Chong EA, et al. Chimeric antigen receptor T cells in refractory B-cell lymphomas. NeW Eng/ J Med 2017;377:2545-54

30. Schuster SJ, Bishop MR, Tam CS, et al. Tisagenlecleucel in adult relapsed or refractory diffuse large B-cell lymphoma. New Eng/ 」 Med. 2019:380:45-56.

31. Abramson JS, Palomba ML, Gordon LI, et al. Lisocabtagen maraleucel for patients with relapsed or refractory large B-cell lymphomas (TRANSCEND NHL 001): a multicentre seamles design study. Lancet. 2020;396:839-52.

32. Wang M, Munoz J, Goy A, et al. KTE-X19 CAR T-cell therapy in relapsed or refractory mantle-cell lymphoma. New Eng/ I Med. 2020;382:1331-42.

33. Maus MV, Alexander S, Bishop MR, et al. Society for Immunotherapy of Cancer (SITC) clinical practice guideline on immune effector cell-related adverse events. I Immunother Cancer. 2020;8:e001511.

34. Khongorzul P, Ling CJ, Khan FU, et al. Antibody-drug conjugates: a comprehensive review. Mol Cancer Res. 2020;18:3-19.

35. Younes A, Kim S, Romaguera J, et al. Phase I multidoseescalation study of the anti-CD19 maytansinoid immunoconjugate SAR3419 administered by intravenous infusion every 3 weeks to patients with relapsed/refractory B-cell lymphoma. J Clin Oncol. 2012;30:2776-82.

36. Coiffier B, Thieblemont C, Guibert S, et al. A phase II, single-arm, multicentre study of coltuximab ravtansine (SAR3419) and rituximab in patients with relapsed or refractory diffuse large Bcell lymphoma. Brit J Haematol. 2016;173:722-30.

37. Walter RB. Expanding use of CD33-directed immunotherapy Expert Opin Biol Ther. 2020;20:955-8.

38. Hicks SW, Tarantelli C, Wilhem A, et al. The novel CD19-targeting antibody-drug conjugate huB4-DGN462 shows improved antitumor activity than SAR3419 in CD19-positive Iymphoma and leukemia models. Haematologica. 2019:104:1633-9.

39. Torsten H, Aniko P, Christoph M, et al. Abstract 62: CD19 - a potential target for amanitin-based ADCs. Exp Mol Ther. 2017;77:62.

40. Tiberghien AC, Levy J-N, Masterson LA, et al. Design and synthesis of tesirine, a clinical antibody-drug conjugate pyrrolobenzodiazepine dimer payload. ACS Med Chem Lett. 2016;7:983-7.

41. Jeffrey SC, Burke PJ, Lyon RP, et al. A potent anti-CD70 antibodydrug conjugate combining a dimeric pyrrolobenzodiazepine drug with site-specific conjugation technology. Bioconjug Chem. 2013;24:1256-63.

42. Hartley JA, Flynn MJ, Bingham JP, et al. Pre-clinical pharmacology and mechanism of action of SG3199, the pyrrolobenzodiazepine (PBD) dimer warhead component of antibody-drug conjugate (ADC) payload tesirine. Sci Rep. 2018;8:10479.

43. Zammarchi F, corbett S, Adams L, et al. ADCT-402, a PBD dimercontaining antibody drug conjugate targeting CD19-expressing malignancies. Blood. 2018:131:1094-105.

44. Kahl BS, Hamadani M, Radford J, et al. A Phase I study of ADCT402 (loncastuximab tesirine), a novel pyrrolobenzodiazepine- based antibody-drug conjugate, in relapsed/refractory B-cell non-Hodgkin lymphoma. Clin Cancer Res. 2019;25:6986-94.

45. Hamadani M, Radford J, Carlo-Stella C, et al. Final results of a phase 1 study of loncastuximab tesirine in relapsed/refractory B-cell non-Hodgkin lymphoma. Blood. 2021;137:2634-45.

46. Carlo-Stella C, Luigi Zinzani PL, Kahl BS, et al. Interim futility analysis of a Phase 2 study of loncastuximab tesirine, a nove pyrrolobenzodiazepine-based antibody-drug conjugate, in patients with relapsed or refractory diffuse large B-cel lymphoma. Blood 2019;134(Suppl. 1):757.

47. Caimi PF, Ai W, Alderuccio JP, et al. Loncastuximab tesirine in relapsed or refractory diffuse large B-cell lymphoma (LOTIS-2): a multicentre, open-label, single-arm, phase 2 trial. Lancet Oncol. 2021;22:790-800

48. Depaus J, Wagner-Johnston N, Zinzani PL, et al. Clinical activity of loncastuximab tesirine plus ibrutinib in non-Hodgkin Iymphoma: updated LOTIS 3 phase 1 results. Hematol Oncol. 2021:39(Suppl. 2). DOl:10.1002/hon.150 2880.

49. ClinicalTrials.gov. Study to Evaluate Loncastuximab Tesirine with Rituximab Versus Immunochemotherapy in Participants
with Relapsed or Refractory Diffuse Large B-cell Lymphoma (LOTIS 5). ClinicalTrials.gov Identifier: NCT04384484. Available (LOTIS 5). ClinicalTrials.gov Identifier: NCTO4384484. Avallab at: clinicaltrials.go
December 2021).

50. ClinicalTrials.gov. Loncastuximab Tesirine in Combination with Rituximab in Patients with Relapsed or Refractory Follicular Lymphoma. ClinicalTrials.gov Identifier: NCT04998669. Available at: clinicaltrials.gov/ct2/show/NCT04998669 (accessed 7 December 2021)

51. ClinicalTrials.gov. Study to Evaluate the Efficacy and Safety of Loncastuximab Tesirine Versus Idelalisib in Participants with Relapsed or Refractory Follicular Lymphoma (LOTIS 6). ClinicalTrials.gov Identifier: NCT04699461. Available at: clinicaltrials. gov/ct2/show/NCT04699461 (accessed 7 December 2021).

52. ClinicalTrials.gov. A Study to Evaluate the Safety and Anticancer Activity of Loncastuximab Tesirine in Combination with Other Anti-cancer Agents in Participants with Relapsed or Refractory B-cell Non-Hodgkin Lymphoma (LOTIS 7). ClinicalTrials.gov Identifier: NCT04970901. Available at: www.clinicaltrials.gov/ct2/show/NCT04970901 (accessed 7 December 2021)

53. ClinicalTrials.gov. A Study to Evaluate the Tolerability, Safety, Pharmacokinetics, and Antitumor Activity of Loncastuximab Tesirine in Combination with Rituximab, Cyclophosphamide Doxorubicin, Vincristine, and Prednisone (R-CHOP) in Participants with Previously Untreated Diffuse Large B-cell Lymphoma (LOTIS-8) (LOTIS-8). ClinicalTrials gov Identifier: NCT04974996. Available at: clinicaltrials.gov/ct2/show/ NCT04974996 (accessed 7 December 2021).

54. Thapa B, Caimi PF, Ardeshna KM, et al. CD19 antibody-drug conjugate therapy in DLBCL does not preclude subsequent responses to CD19-directed CAR T-cell therapy Blood Adv. 2020;4:3850-2. 